\section{Periprocedural Management of Patients on Dabigatran Etexilate Treatment}

Dabigatran etexilate is a direct thrombin inhibitor that was initially introduced for oral anticoagulation for the prevention or treatment of venous thrombosis. ${ }^{1}$ After the recently concluded phase III trial Randomized Evaluation of Long-Term Anticoagulation Therapy, ${ }^{2}$ dabigatran has been approved by the US Food and Drug Administration as a potential replacement for warfarin in patients with nonvalvular atrial fibrillation to reduce the risk of stroke and systemic embolism. Dabigatran was administered, in a blinded fashion, in capsules containing either 110 or $150 \mathrm{mg}$ of the drug, to be taken twice daily.

With the anticipated increase in dabigatran use, neurointerventionalists will face the challenge of making treatment decisions in patients on dabigatran. Patients with therapeutic levels of dabigatran who undergo invasive procedures including endovascular interventions are at increased risk for bleeding. Elective neurointerventional procedures may require the temporary discontinuation of dabigatran etexilate, depending on the degree of renal impairment and risk of bleeding. ${ }^{3}$ Because renal impairment may prolong the half-life of dabigatran, it is important to check the creatinine clearance (CCr) before making treatment decisions.

The current evidence supports discontinuing dabigatran 24 hours before an interventional procedure in patients with normal renal function $(\mathrm{CCr}>50 \mathrm{~mL} / \mathrm{min})$ and a standard bleeding risk. Dabigatran should be discontinued 2-4 days before interventional procedures in patients with normal renal function and a high bleeding risk in whom complete hemostasis may be required (ie, spinal puncture or placement of a spinal or epidural catheter or port). Dabigatran should be discontinued at least 2 days before an interventional procedure in patients with impaired renal function $(\mathrm{CCr}<50 \mathrm{~mL} / \mathrm{min}$ ) and a standard bleeding risk. In patients with impaired renal function and at higher risk of bleeding, dabigatran should be stopped 4 days before the procedure.

Management of patients on dabigatran who require emergent neurointerventional procedures is a more challenging issue. Unlike warfarin, the therapeutic effect of dabigatran cannot be reliably measured by using the international normalized ratio. Due to reliable pharmacodynamic and pharmacokinetic properties, routine coagu- lation tests are not necessary to monitor the effect of dabigatran. However, there is a need to quantitate the intensity of anticoagulation activity of dabigatran in such settings. Other tests such as the thrombin time, activated partial thromboplastin time (aPTT), and ecarin clotting time have been reported to correlate with dabigatran serum concentration. $^{3}$

The Interventional Management of Stroke (IMS) III trial ${ }^{4}$ proposed that administration of tissue plasminogen activator or endovascular intervention could be considered in patients at 48 hours after last intake of dabigatran, or within 48 hours of the last intake and with normal aPTT in the same way as heparin. Because no guidelines exist on how to monitor the therapeutic activity effect of dabigatran accurately, emergent neurointerventional procedures in dabigatrantreated patients should be approached cautiously. The time interval between the last dose administration and the neurointerventional procedure appears to be the most reliable criterion for assessing the risk of bleeding events. At this time, we consider the criteria of IMS III acceptable but recommend ongoing evaluation of practices.

\section{References}

1. Siddiqui FM, Qureshi AI. Dabigatran etexilate, a new oral direct thrombin inhibitor, for stroke prevention in patients with atrial fibrillation. Expert Opin Pharmacother 2010;11:1403-11

2. Connolly SJ, Ezekowitz MD, Yusuf S, et al. Dabigatran versus warfarin in patients with atrial fibrillation. $N$ Engl J Med 2009;361:1139-51

3. van Ryn J, Stangier J, Haertter S, et al. Dabigatran etexilate: a novel, reversible, oral direct thrombin inhibitor-interpretation of coagulation assays and reversal of anticoagulant activity. Thromb Haemost 2010;103:1116-27. Epub 2010 Mar 29

4. CinicalTrials.gov. Interventional Management of Stroke (IMS) III Trial (IMS III). http://clinicaltrials.gov/ct2/show/NCT00359424. Accessed September 23, 2011

M. Watanabe

A. I. Qureshi

Zeenat Qureshi Stroke Research Center University of Minnesota

Minneapolis, Minnesota

F. M. Siddiqui

Department of Neurology

Southern Illinois University Health Care

Springfield, Illinois

http://dx.doi.org/10.3174/ajnr.A2731 